Mon. Not. R. Astron. Soc. 000, 000-000 (0000) Printed 15 October $2018 \quad$ (MN LATEX style file v2.2)

\title{
Discovery of a WO star in the Scutum-Crux arm of the inner Galaxy
}

\author{
Janet E. Drew ${ }^{1}$, M. J. Barlow ${ }^{2}$, Y. C. Unruh ${ }^{1}$, Q. A. Parker ${ }^{3,4}$, R. Wesson ${ }^{2}$, \\ M. J. Pierce ${ }^{5}$, M. R. W. Masheder ${ }^{5} \&$ S. Phillipps ${ }^{5}$ \\ ${ }^{1}$ Imperial College of Science, Technology and Medicine, Blackett Laboratory, Exhibition Road, London, SW7 2AZ, U.K. \\ ${ }^{2}$ University College London, Department of Physics 8 Astronomy, Gower Street, London WC1E 6BT, U.K. \\ ${ }^{3}$ Department of Physics, Macquarie University, NSW 2109, Australia \\ ${ }^{4}$ Anglo-Australian Observatory, PO Box 296, Epping NSW 1710, Australia \\ ${ }^{5}$ Astrophysics Group, Department of Physics, Bristol University, Tyndall Avenue, Bristol, BS8 1TL, U.K.
}

received, accepted

\begin{abstract}
We report the discovery of only the fourth massive WO star to be found in the Milky Way, and only the seventh identified within the Local Group. This has resulted from the first observations made in a programme of follow-up spectroscopy of candidate emission line stars from the AAO/UK Schmidt Southern Galactic Plane $\mathrm{H} \alpha$ Survey. The optical spectrum of this star, to become WR 93b in the Catalogue of Galactic Wolf-Rayet stars, is presented and described. WR 93b is classified as WO3 and is shown to be highly reddened $\left(E_{B-V}=2.1 \pm 0.1\right)$. A recombination line analysis of the emission lines yields the abundance ratios $\mathrm{C} / \mathrm{He}=0.95$ and $\mathrm{O} / \mathrm{He}=0.13$ (by number). Comparisons at near infrared wavelengths of reddening corrected photometry between WR 93b and both of Sand 2 (WO3, $D=49 \mathrm{kpc}$ ) and Sand 5 (WO2, $D=1.75 \mathrm{kpc}$ ) yields a consistent distance to WR 93b of $3.4 \mathrm{kpc}$. Positioned at Galactic co-ordinates $\ell=353.27^{\circ}, b=-0.85^{\circ}$, the star is most likely located in the Scutum-Crux Arm of the inner Milky Way. We note that none of the four Galactic WO stars lies significantly beyond the Solar Circle (with two well inside).

Estimation of the wind terminal velocity in WR $93 \mathrm{~b}$ at $5750 \mathrm{~km} \mathrm{~s}^{-1}$ makes this star the current wind speed record holder among all non-degenerate stars.
\end{abstract}

Key words: surveys - stars: Wolf-Rayet - stars: individual: WR 93b - stars: distances - Galaxy: stellar content

\section{INTRODUCTION}

In this paper we present the discovery of a particularly rare type of emission line star made in the initial phase of a longterm spectroscopy programme which aims to confirm and provide preliminary classification of spatially-unresolved line-excess objects contained within the AAO/UK Schmidt Southern Galactic Plane $\mathrm{H} \alpha$ Survey (Parker et al 2003). The star in question has been revealed as only the fourth massive WO star to be found in the Galaxy - bringing the total identified within the Local Group to just seven. When it is assimilated into the next revision of the catalogue of massive Wolf-Rayet stars in the Galaxy, compiled and maintained by van der Hucht (2001), its position in the sky will earn it the designation WR 93b. Hereafter we refer to this newly discovered star by this name. The WO stars are the most chemically extreme Wolf-Rayet (WR) stars, whose spectra are dominated by high-excitation oxygen and carbon lines.
Objects in this elite group of stars are viewed as plausible progenitors for extreme, chemically-peculiar Type Ib/c supernovae (Woosley, Heger \& Weaver 2002) and some GRBs (e.g. Schaefer et al 2003).

The history of this presently rare type of object begins with the last generation of galactic emission line surveys: Sanduleak (1971) presented a list of five WR stars, two in the Magellanic Clouds, which had strong O vi 3811,34 A emission features that had previously been found only amongst planetary nebula nuclei. Barlow \& Hummer (1982) proposed that one of these five stars was indeed a PN central star but that the other four corresponded to an advanced stage of evolution of massive stars, beyond the WC phase, and classified them as WO Wolf-Rayet stars. Since this time, two more have been added to this grouping (MS 4, by Smith, Shara \& Moffat 1990 and DR 1 in the dwarf irregular galaxy IC 1613 by Kingsburgh \& Barlow 1995). In general terms, discoveries of Galactic WR stars have been assisted greatly by opti- 
cal objective-prism surveys (Stephenson \& Sanduleak 1971, MacConnell \& Sanduleak 1970) and narrow-band imaging surveys (most recently, Shara et al. 1999). The result we present here uses the latter technique in the red part of the spectrum, and serves as an encouragement that much remains to be discovered.

In the next section we introduce the AAO/UK Schmidt Southern Galactic Plane $\mathrm{H} \alpha$ Survey and describe the data it contains relevant to the newly-discovered WO star and its locale. We then describe how the WO star came to be included in our initial programme of follow-up spectroscopy using the AAO/UK Schmidt multi-fibre spectroscopy facility, 6dF. This is followed, in Section 3, by presentation of both the $6 \mathrm{dF}$ data obtained in May/June 2003 (§3.1), and flux-calibrated long-slit spectra obtained at the William Herschel Telescope (WHT) in August 2003 (§3.2). We are then in a position to classify the WO star and determine its reddening $(\S 4)$. We also present estimates of $\mathrm{C} / \mathrm{He}$ and $\mathrm{O} / \mathrm{He}$ abundance ratios deduced from the emission lines ( $(5)$. Finally, by comparison with other WO stars at known distances, we derive the distance to WR 93b ( $\S 6)$ : at $3.4 \mathrm{kpc}$, and along a line of sight passing within a few degrees of the Galactic Centre, it seems likely the new WO star is associated with the Scutum-Crux spiral arm (Russeil 2003) without a doubt it is well inside the Solar Circle. We close in $\S 7$ with comment on the 4 -strong group of galactic WO stars and on the prospects for future discoveries.

\section{UKST H $\alpha$ SURVEY IMAGING OBSERVATIONS}

The AAO/UKST narrow-band $\mathrm{H} \alpha$ Survey of the Southern Galactic Plane and Magellanic Clouds is the last photographic sky survey to have been carried out on the UK Schmidt Telescope (UKST). It has recently been completed (Parker et al. 2003) and is now available as digital survey data derived from SuperCOSMOS scans of the original survey films at $10 \mu \mathrm{m}(0.67$ arcsec $)$ resolution (hereafter, the SHS database, located at http://wwwwfau.roe.ac.uk/sss/halpha/). This survey used a specially designed, $70 \AA$ bandpass, $\mathrm{H} \alpha$ interference filter (Parker \& Bland-Hawthorn 1998). When combined with fine-grained Tech-Pan emulsion as detector (Parker and Malin, 1999), it made possible a survey of Galactic ionised hydrogen that is a powerful combination of depth, spatial resolution and areal coverage (Parker \& Phillipps, 1998). Each field observed has an effective dimension projected on the sky of $4^{\circ} \times 4^{\circ}$. The survey policy was to gather contemporaneous 15-minute broad-band 'short $R$ ' exposures alongside 3-hour narrow-band $\mathrm{H} \alpha$ exposures in order to yield exposure pairs, well matched both in terms of sensitivity limit $(R=19.5)$ for continuum point sources and in terms of point-spread functions. The entire southern plane of the Milky Way has been imaged within the Galactic latitude range $-10^{\circ}<b<+10^{\circ}$ in this way. These exposure pairs provide the source material for a variety of continuing research projects (see e.g. Morgan, Parker \& Russeil 2001; Parker \& Morgan 2003; Parker et al 2003).

We have embarked on a programme aimed at providing spectroscopic confirmations of spatially-unresolved candidate emission line stars derived from the SHS database.

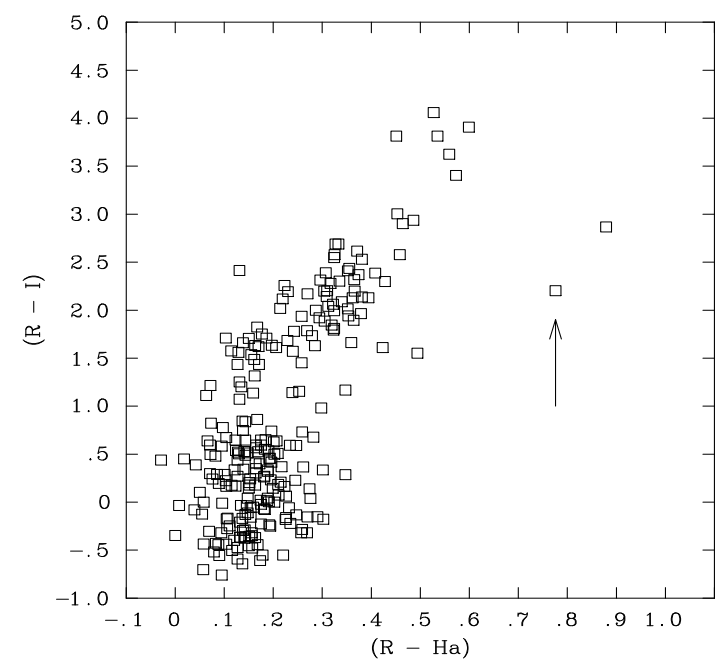

Figure 1. A plot of $(R-I)$ versus $(R-H \alpha)$ for all SHS catalogue point sources with $14.5<R<16.0$, lying within a 15 arcmin radius of WR 93b. The WO star itself is indicated by the arrow. Note that most stars in this diagram congregate in the rectangle defined by $(R-I)<2$ and $-0.1<(R-H \alpha)<0.3$ : the stars trailing off in a lightly sloping line to higher $(R-I)$ and $(R-$ $H \alpha$ ) are mainly extreme red stars with prominent molecular band structure in the red part of the spectrum. Interstellar reddening will displace a star vertically in this diagram. For the most part, the SHS database is calibrated to yield $(R-H \alpha) \simeq 0$ in the mean - here, in this small field, there happens to be an offset to between 0.1 and 0.2 in this colour.

This began in 2003 with limited data-taking using the UKST $6 \mathrm{dF}$ facility to follow up a few fields in the brighter half of the available magnitude range $(12.5<R<16)$, with a view to establishing observing procedures for the longer term. The broad goal of the programme is to increase by an order of magnitude Galactic samples of the relatively short-lived, rare phases of stellar evolution typified by strong line emission at $\mathrm{H} \alpha$. This is expected to be achieved by dropping the current typical limit of $R \sim 13$ on survey samples of emission line stars (cf. MacConnell 1982, Robertson \& Jordan 1989) down to $R \sim 19$. The value in this is that several groups of young and evolved objects, critical to our understanding of pre- and post-main-sequence stellar evolution, are currently extremely poorly sampled: as noted already, the massive WO star reported here is only the fourth to be found in the Milky Way.

As part of our initial spectroscopy programme, we chose to observe targets drawn from field HAL0555 within the magnitude range $14.5<R<16.0$. Centred at $17 \mathrm{~h} 20 \mathrm{~m}$ $-36^{\circ}$ (1950 co-ordinates), this field is a highly reddened one straddling the Galactic equator, just a few degrees in longitude away from the Galactic Centre. 106 point sources were selected for spectroscopy from a longer list of candidate stars with relatively extreme $\mathrm{H} \alpha$ magnitude excesses with respect to the associated short-red broadband magnitudes $((R-H \alpha)>0.6$, with the great majority of stars falling in the range $-0.2<(R-H \alpha)<0.5)$. Note that the SHS $\mathrm{H} \alpha$ magnitudes are derived from calibration of the $\mathrm{H} \alpha$ images against $R$ standards, and undergo final adjustments as a function of $R$ magnitude such that the median $(R-H \alpha)$ 

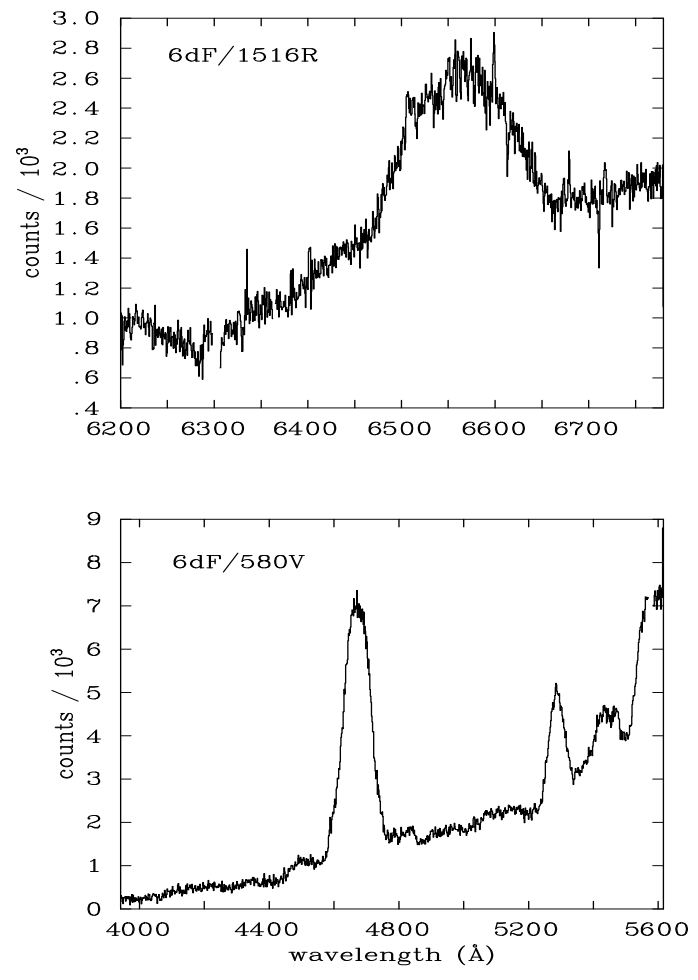

Figure 2. The spectra of WR 93b obtained in May/June 2003 using $6 \mathrm{dF}$. The top panel contains the spectrum obtained using the $1516 \mathrm{R}$ grating in the ' $\mathrm{H} \alpha$ ' region (for this star, the emission at $\sim 6560 \AA$ is a HeII/Civ blend). The bottom panel shows the broader coverage $580 \mathrm{~V}$ spectrum.

colour for any given field is set to zero (cf. the discussion of calibration of the SuperCOSMOS Sky Survey presented in Hambly et al 2001). The plan for the spectroscopy, in the long term, is to also select on $(R-I)$ in order to reduce the number of unwanted red stars whose molecular band structure mimics $\mathrm{H} \alpha$ excess. However this was not done here so that we might determine empirically how best to apply such a cut.

One of the sources selected for spectroscopy was located in the dark NE part of the field at RA 1732 03.30, Dec -35 0432.5 (J2000) and was listed in the SHS database as having $R=14.672$, and $(R-H \alpha)=0.776$ (see Hambly et al 2001 for a discussion of likely photographic errors). The suitability of this object for follow-up spectroscopy becomes apparent on considering its position in a magnitude-limited plot of point-source $(R-I)$ colour versus $(R-H \alpha)$ excess for objects in its immediate locality. In Figure 1 we present such a plot for all stars within 15 arcmin of the WO star: it can be seen there that it is one of just two objects sitting in relative isolation, on the 'excess' side of a well-populated locus of stars. The other clear excess object star still awaits spectroscopic follow-up.

\section{SPECTROSCOPIC OBSERVATIONS}

\subsection{UKST/6dF Observations}

Prompted as described above, we used the AAO/UK Schmidt $6 \mathrm{dF}$ multi-fibre spectrograph to obtain spectra of 106 spatially-unresolved objects located in field 555 of the imaging survey: every target selected satisfied the constraints $14.5<R<16$ and $(R-H \alpha)>0.6$. This target list was observed first using the $1516 \mathrm{R}$ grating on 2003 May 24, and for a second time on 2003 June 6 using the $580 \mathrm{~V}$ grating (see Table 1 for details). The respective spectral resolutions of the two gratings are $\sim 2 \AA$ and $\sim 6 \AA$. The $1516 \mathrm{R}$ observations were performed as a series of alternating on-target and sky-offset observations, to ensure the best possible subtraction of diffuse $\mathrm{H} \alpha$ emission by sampling nebulosity in the immediate environment of every target star.

Both datasets were extracted from the CCD frames using the $6 \mathrm{dF}$ adaptation of the $2 \mathrm{dfdr}$ software package (see http://www.aao.gov.au/AAO/2df/manual.htm). The extraction consisted of: bias subtraction using the overscan strip; flat field extraction; fibre-by-fibre arc extraction and calibration; optimal extraction of the observed spectrum from each fibre, followed by flat-field correction and wavelength calibration. For the May 1516R observations, the sky offset frames were each subtracted from the associated target frames and then combined, weighted according to their statistical quality, to give a single reduced frame containing up to 150 spectra each of 1032 wavelength-calibrated pixels. No throughput correction was applied to these data. The June $580 \mathrm{~V}$ observations were handled differently in that a fibre throughput correction was applied so as to enable sky subtraction using the mean spectrum of the designated sky fibres.

The extracted 6dF spectra of WR 93b are shown in Figure 2. It was immediately evident from these data that the object was a Wolf-Rayet star presenting extremely broad emission features. Furthermore it was possible to note the partial capture of the Ovi $\lambda 6200$ line in the $1516 \mathrm{R}$ observation (Figure 2, top panel) and the clear presence of Ovi $\lambda 5290$ in the $580 \mathrm{~V}$ observation (Figure 2, lower panel). Either of these features at the observed prominence is sufficient to identify the object as a WO star. It was also evident from both spectra, when considered in comparison to those of other targets, that WR 93b must be significantly reddened. In order to obtain the extended blue coverage of the optical spectrum needed for WO sub-type determination (using ratios of emission line equivalent widths) and to assess the relative spectral energy distribution, it was decided to obtain additional observations using ISIS on the William Herschel Telescope.

\subsection{WHT/ISIS observations}

Data were obtained on 2003 August 2nd using the twinarmed ISIS spectrograph mounted on the William Herschel Telescope, located in La Palma. It was a clear night of good seeing. Because WR 93b is far south relative to the latitude of the Isaac Newton Group on La Palma $\left(+28^{\circ}\right)$, care was taken to time the observations to within minutes of meridian transit, setting the slit angle to the north-south position so as to minimise slit losses caused by atmospheric dispersion.

The spectrograph setup included the $5400 \AA$ dichroic such that the blue arm, with R600B grating, delivered a reliable spectrum over the range 3600-5000 $⿱$; whilst the red arm with R316R grating gave good results over the range 5500-7900 $\AA$. We do not use data in the range 5000-5500 $\AA$ as these are visibly affected by the dichroic transmission cut. 
Table 1. Abbreviated log of observations

\begin{tabular}{|c|c|c|c|c|c|c|c|}
\hline $\begin{array}{l}\text { telescope/ } \\
\text { spectrograph }\end{array}$ & $\begin{array}{l}\text { observation } \\
\text { date }\end{array}$ & UT start & grating & $\begin{array}{l}\text { central } \\
\text { wavelength } \\
(\AA)\end{array}$ & $\begin{array}{r}\text { exposures } \\
n \times t \\
(\mathrm{sec})\end{array}$ & $\begin{array}{l}\text { seeing } \\
(\operatorname{arcsec})\end{array}$ & additional information \\
\hline $\mathrm{UKST} / 6 \mathrm{dF}$ & $2003 / 05 / 24$ & $15: 55$ & $1516 \mathrm{R}$ & 6490 & $\begin{array}{l}5 \times 1200 \\
5 \times 1200\end{array}$ & $1-2$ & $\begin{array}{l}\text { interlaced offsets (RA,Dec arcsec: } \\
+10,+5 ;+10,-5 ;-10,+5 ;-10,-5 \\
+5,-10)\end{array}$ \\
\hline UKST/6dF & 2003/06/06 & $16: 08$ & $580 \mathrm{~V}$ & 4780 & $7 \times 1200$ & $3-4$ & \\
\hline \multirow[t]{4}{*}{ WHT/ISIS } & $2003 / 08 / 02$ & $20: 35$ & R600B & 4282 & $2 \times 120$ & 1 & $\begin{array}{l}\text { HZ } 44 \text { flux standard } \\
\text { airmass } 1.22,1.23 \text { slit } 8.0 \text { arcsec } \\
\text { slit PA } 90\end{array}$ \\
\hline & & $20: 35$ & R316R & 6547 & $2 \times 120$ & & HZ 44 flux standard \\
\hline & & $21: 03$ & R600B & 4282 & $2 \times 900$ & & $\begin{array}{l}\text { WO star: airmass } 2.37,2.32 \\
\text { slit } 0.8 \text { arcsec, PA } 0\end{array}$ \\
\hline & & $21: 03$ & R316R & 6547 & $2 \times 900$ & & WO star \\
\hline
\end{tabular}

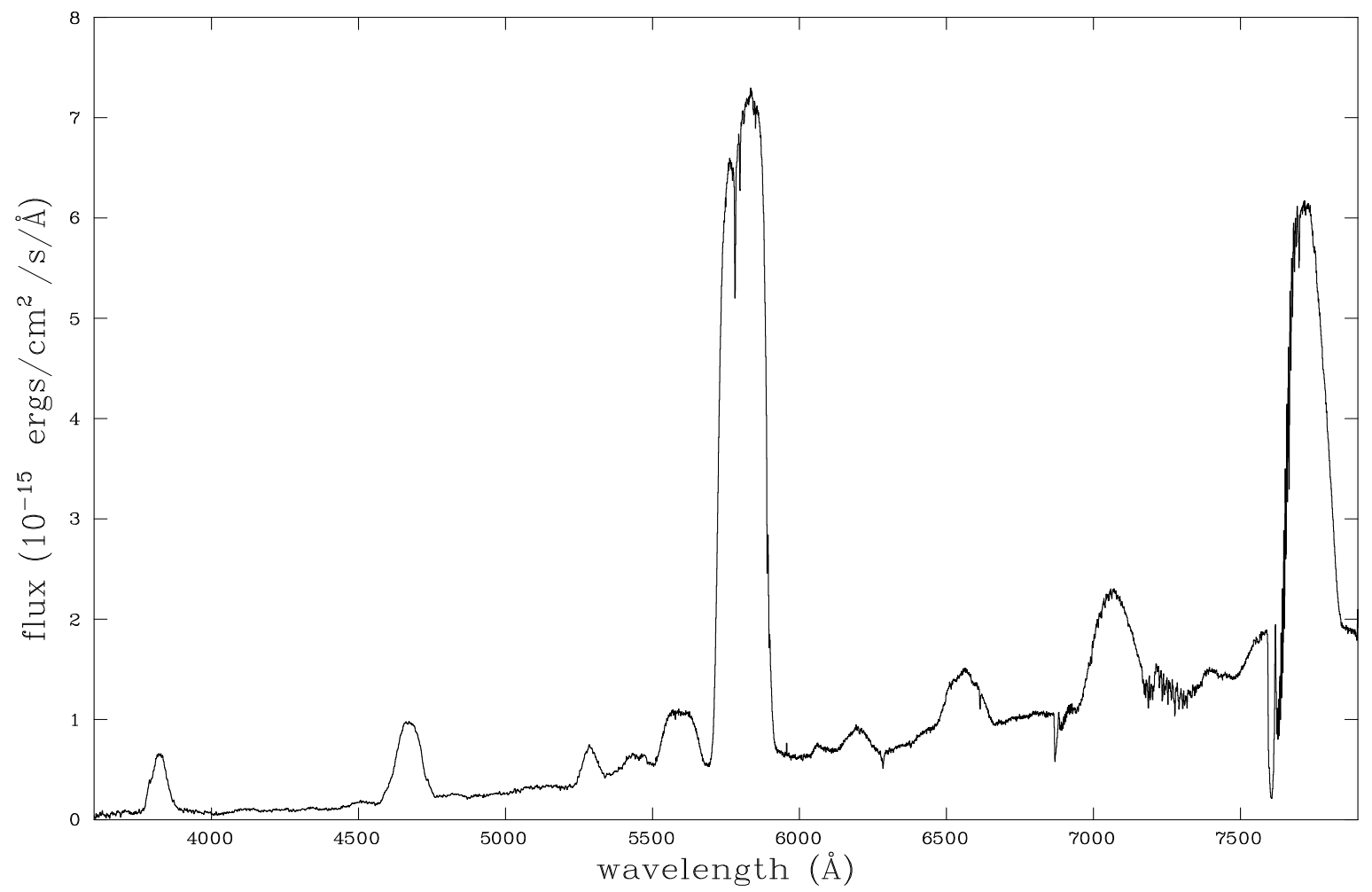

Figure 3. The combined flux-calibrated spectrum of WR 93b obtained at the William Herschel Telescope on 2003 August 2, with the $6 \mathrm{dF} / 580 \mathrm{~V}$ spectrum spliced in to cover 5000-5500 A. Note that the absolute flux level plotted will be an under-estimate because of slit losses.

The respective spectral resolutions obtained were $1.5 \AA$ and $2.8 \AA$, giving e.g. a velocity resolution of $145 \mathrm{~km} \mathrm{~s}^{-1}$ at the CIV $\lambda \lambda 5801,12$ doublet. Further details of the observations are given in Table 1 . Wavelength calibration was performed using arc exposures obtained immediately after the stellar spectra, at the same telescope pointing. Wide-slit observations of the flux standard HZ 44 (Massey et al 1988) obtained a little earlier in the night were used to flux-calibrate the data after extraction to one-dimensional format. Before this calibration was applied to the WO-star spectra, the nec- essarily large correction for air mass was also made. The ISIS spectra as displayed here have been smoothed using a gaussian of FWHM $=1 \AA$ in order to achieve some noise reduction. No correction has been made for telluric absorption.

In Figure 3, we show the flux-calibrated ISIS spectra together with a rescaled interpolation of the UKST/6dF $580 \mathrm{~V}$ spectrum, that usefully spans the 5000-5500 ̊ gap left by the ISIS results. It turns out that this interpolation can be achieved with confidence because there is excellent agree- 
Table 2. Emission line identifications and measurements from the spectrum of WR 93b over the wavelength range 3600-7900 A. Observed wavelengths are centroid wavelengths to the nearest $5 \AA$; line widths (FWHM) are quoted to the nearest $10 \AA$. A colon placed against a numerical value indicates greater uncertainty than for other values listed in the same column. C IV $\lambda 7062$ has some telluric absorption in its extreme red wing. The last three emission lines listed are included for completeness: they were not measured in view of the more severe uncorrected telluric absorption overlapping them. Columns 4, 5 and 6 contain equivalent width (EW) measurements and the quantities derived from them that are needed for the abundance determination presented in $\S 5$. In the last two columns, and for the purpose of comparison with WR 93b, equivalent widths are given for Sand 2 and and Sand 5 (data from KBS95).

\begin{tabular}{|c|c|c|c|c|c|c|c|}
\hline $\begin{array}{l}\lambda_{o b s} \\
(\AA)\end{array}$ & ID & $\begin{array}{l}\text { FWHM } \\
(\AA)\end{array}$ & $\begin{array}{l}\text { EW } \\
(\AA)\end{array}$ & $\begin{array}{l}\mathrm{I} \\
\left(10^{-12} \text { ergs }\right. \\
\left.\mathrm{cm}^{-2} \mathrm{~s}^{-1}\right)\end{array}$ & $\begin{array}{l}\mathrm{I} / \mathrm{Q} \\
\left(\mathrm{cm}^{-5}\right)\end{array}$ & $\begin{array}{l}\text { Comparison } \\
\text { Sand } 2 \\
\text { (WO3) }\end{array}$ & $\begin{array}{l}\mathrm{EW}(\AA) \\
\text { Sand } 5 \\
(\text { WO2) }\end{array}$ \\
\hline 3820 & $\mathrm{O}$ vi $3811,34\left(3 \mathrm{p}^{2} \mathrm{P} \rightarrow 3 \mathrm{~s}^{2} \mathrm{~S}\right)$ & 60 & $670 \pm 100$ & & & $336 \pm 6$ & $1005 \pm 17$ \\
\hline 4120: & $\begin{array}{l}\text { O v } 4119-4178\left(3 \mathrm{p}^{\prime}{ }^{3} \mathrm{D} \rightarrow 3 \mathrm{~s}^{\prime}{ }^{3} \mathrm{P}^{o}\right) \\
\text { O v } 4120-4159\left(3 \mathrm{~d}^{\prime}{ }^{3} \mathrm{P}^{o} \rightarrow 3 \mathrm{p}^{\prime}{ }^{3} \mathrm{~S}\right)\end{array}$ & & $30 \pm 5$ & & & & \\
\hline 4505: & $\begin{array}{l}\text { O vi } 4499(10 \rightarrow 8) \\
\text { O v } 4521(9 \rightarrow 7), 4523\left(3 \mathrm{~d}^{\prime}{ }^{1} \mathrm{P}^{o} \rightarrow 3 \mathrm{p}^{\prime}{ }^{1} \mathrm{D}\right)\end{array}$ & 60: & $23 \pm 5$ & & & & \\
\hline 4670 & $\begin{array}{l}\text { C IV } 4658(6 \rightarrow 5), 4685(8 \rightarrow 6) \\
\text { He II } 4686(4 \rightarrow 3)\end{array}$ & 90 & $460 \pm 25$ & & & $531 \pm 4$ & $496 \pm 7$ \\
\hline 4820: & $?$ & 50: & $10 \pm 5$ & & & & \\
\hline 5285 & O vi $5290(8 \rightarrow 7)$ & 50 & $58 \pm 3$ & 11.0 & $1.05 \times 10^{13}$ & $45 \pm 2$ & $85 \pm 5$ \\
\hline 5440: & $\begin{array}{l}\text { He II } 5411(7 \rightarrow 4), \\
\text { C IV } 5411(14 \rightarrow 8), 5470(10 \rightarrow 7)\end{array}$ & 110: & $67 \pm 5$ & & & $65 \pm 2$ & $74 \pm 5$ \\
\hline 5590 & O v $5590\left(3 d^{3} \mathrm{D} \rightarrow 3 \mathrm{p}^{3} \mathrm{P}^{o}\right)$ & 130 & $170 \pm 7$ & 27.4 & $6.3 \times 10^{12}$ & $112 \pm 3$ & $222 \pm 10$ \\
\hline 5805 & $\mathrm{C}$ IV $5801,12\left(3 \mathrm{p}^{2} \mathrm{P}^{o} \rightarrow 3 \mathrm{~s}^{2} \mathrm{~S}\right)$ & 160 & $1940 \pm 30$ & 278 & $2.00 \times 10^{14}$ & $2450 \pm 190$ & $374 \pm 10$ \\
\hline 6070 & $\begin{array}{l}\text { O VIII } 6068(10 \rightarrow 9), 6064(13 \rightarrow 11) \\
\text { O vil } 6085(12 \rightarrow 10)\end{array}$ & 60 & $9 \pm 3$ & 0.87 & $\begin{array}{l}4.3 \times 10^{11} \\
4.3 \times 10^{11}\end{array}$ & & \\
\hline 6195 & O vi $6200(11 \rightarrow 9,13 \rightarrow 11)$ & 80 & $28 \pm 4$ & 3.3 & $1.08 \times 10^{13}$ & $12 \pm 2$ & $40 \pm 4$ \\
\hline 6440 & $\mathrm{O} \vee 3 \mathrm{~d}^{\prime} \rightarrow 3 \mathrm{p}^{\prime}$ & 70 & $9 \pm 3$ & & & & \\
\hline 6555 & $\begin{array}{l}\text { He II } 6560(6 \rightarrow 4) \\
\text { C IV } 6560(12 \rightarrow 8)\end{array}$ & 120 & $85 \pm 7$ & 8.5 & $\begin{array}{l}1.42 \times 10^{14} \\
\left(1.35 \times 10^{14}\right)\end{array}$ & $93 \pm 3$ & $182 \pm 5$ \\
\hline 7065 & $\mathrm{C}$ IV $7062(9 \rightarrow 7)$ & & $140 \pm 15$ & 11.2 & $1.35 \times 10^{14}$ & $102 \pm 5$ & $96 \pm 8$ \\
\hline 7390 & O v? $(8 \rightarrow 7$ low $\ell ?)$ & & weak & & & & \\
\hline 7590 & $\mathrm{O} \vee 7592,7611(8 \rightarrow 7)$ & & intermediate & & & & \\
\hline 7725 & $\begin{array}{l}\text { C IV } 7726(7 \rightarrow 6), 7736(11 \rightarrow 8), \\
\text { O VI } 7717(9 \rightarrow 8)\end{array}$ & & very strong & & & & \\
\hline
\end{tabular}

ment between the UKST and WHT observations in terms of both emission line contrast and profile shape where the two overlap. This is reassuring as we would not expect, a priori, the WO-star line spectrum to vary between the two epochs of observation.

\section{THE SPECTRAL TYPE, WIND TERMINAL VELOCITY OF, AND REDDENING TOWARDS WR 93B}

The outstanding properties of the merged flux-calibrated spectrum (Figure 3) are the red slope of the continuum and the breadth and extraordinary prominence of the strongest emission lines. For example, the continuum flux rises by a factor of $\sim 10$ between $4000 \AA$ and $\sim 5700 \AA$, whilst the CIV $\lambda \lambda 5801,12$ emission stands, at its peak, at $\sim 13$ times the continuum level (with an equivalent width of $\sim 1900 \AA$ A!). Observed wavelengths, likely identifications, FWHM estimates and equivalent width measurements for the emission features seen in the spectrum are laid out in Table 2.

In order to assign a spectral type to WR 93b, we refer to the classification criteria given by Crowther, de Marco and Barlow (1998). The primary discriminant is the ratio between the equivalent widths (EWs) of the O VI $\lambda \lambda 3811,34$ and $\mathrm{O} v \lambda \lambda 5590$ features: here this comes out at $3.9 \pm 0.6$, close to but just under the WO2/WO3 boundary value of 4 (see data in Table 2, and Table 3 of Crowther et al 1998). The secondary classification criteria are the $(\mathrm{O}$ VI $\lambda \lambda 3811,34 / \mathrm{C}$ IV $\lambda \lambda 5801,12)$ and (O VII $\lambda 5670 / \mathrm{OV} \lambda 5590)$ equivalent width ratios. The value of the first of these is $0.35 \pm 0.05$, whilst the second ratio can only be described as small since the $\mathrm{O}$ VII $\lambda 5670$ cannot be said to have been definitely detected. These indicators are more clearly in line with a WO3 classification, which we prefer for the time being.

This provisional classification places the new WO star in the same sub-type as the LMC WO star, Sand 2. This similarity is reinforced on making a broader comparison between the equivalent width data (Table 2) given for WR 93b and reproduced for Sand 2 and the Galactic WO2 star, Sand 5, from Kingsburgh, Barlow \& Storey (1995, hereafter KBS95). We can conclude that WR 93b exhibits slightly higher excitation than Sand 2, whilst certainly not being as extreme as Sand 5. In due course, a more decisive spectral classification may become possible via a measurement of the relative equivalent widths of O IV $\lambda 3400\left(3 \mathrm{~d}{ }^{2} \mathrm{D}-3 \mathrm{p}{ }^{2} \mathrm{P}^{o}\right)$ and $\mathrm{O}$ VI $\lambda \lambda 3434,38(7-6,11-8)$ : for the WO3 stars, Sand 1 and Sand 2, KBS95 found $\mathrm{EW}(\mathrm{O}$ IV) $>\mathrm{EW}(\mathrm{O}$ VI) whilst for the WO2 stars Sand 4 and Sand 5, the O IV line was undetectable.

To estimate the terminal stellar wind velocity, we measure the full width at zero intensity (FWZI) of the C IV $\lambda \lambda 5801,12$ doublet. We adopt the same approach to this as did KBS95. For the case of the SMC WO3 star, Sand 1, 
they showed that the velocity corresponding to half the FWZI of this bright optical line (after correction for the $10.65 \AA$ doublet separation) matched closely the terminal wind velocity measured from the black absorption edge of the C IV 1548,51 $\AA$ resonance doublet in a high resolution $I U E$ spectrum. Respectively, these velocities were 4150 and $4200 \mathrm{~km} \mathrm{~s}^{-1}$. For Sand 2, KBS95 derived a velocity of $4450 \mathrm{~km} \mathrm{~s}^{-1}$ from the C IV 5801,12 $\AA \mathrm{FWZI} / 2$, after correction for the doublet separation. Crowther et al. (2000) subsequently obtained $H S T$ ultraviolet spectra of Sand 2 which yielded a wind terminal velocity of $4100 \mathrm{~km} \mathrm{~s}^{-1}$ from the black absorption edge of the C IV $\lambda \lambda 1548,51$ doublet. For WR 93b, the measured FWZI of the C IV 5801,12 $\AA$ doublet is $233.4 \AA$. After taking off $10.65 \AA$ for the doublet separation, this feature's FWZI/2 yields a wind terminal velocity of $5750 \mathrm{~km} \mathrm{~s}^{-1}$ - somewhat higher than the $5500 \mathrm{~km} \mathrm{~s}^{-1}$ measured by KBS95 for the Galactic WO2 star Sand 5. This is the highest wind terminal velocity so far determined for any star. Certainly the measured FWHM of this very bright line $(160 \AA)$ is larger for WR 93b than for any of the other WO stars.

Given that the star is a WR star, it is evident that we should expect its intrinsic SED to be very blue. In this spirit, KBS95 estimated WO star reddenings using the finding that a wide range of Wolf-Rayet sub-types yield optical SEDs closely following the power law $F_{\lambda} \propto \lambda^{-3}$ (Morris et al 1993). In the specific instances of Sand 2 in the LMC and also Sand 5, both with independent measures of reddening, KBS95 were able to demonstrate the utility of this approach by finding that the dereddened SEDs did indeed fit with expectation (the best fit indices were respectively -3.04 and -3.02). Accordingly, for WR 93b, we identify the range in $E(B-V)$ that restores the optical SED most nearly to a $\lambda^{-3}$ power law: in the case of a standard Galactic reddening law with $R=3.1$, this is $2.0<E(B-V)<2.35$, or equivalently $6.1<A_{V}<7.2$.

Confidence in the reddening estimate grows on noting that corrections consistent with the deduced range have the added virtue of yielding convincingly flat-topped C IV $\lambda \lambda 5801,12$ profile. Before correction (Figure 3 ), there is a very marked red skew of the peak flux in the profile. Such a strong line, formed by a relatively lowly-ionised species in the context of these stellar winds, can be presumed to sample the terminal flow very well. The profile of such a line would be expected to have a reflection-symmetric, nearly 'rectangular', appearance (interpolating across the tops of the superposed narrow interstellar absorption lines). In fact, we find that the reflection symmetry is most perfect for $E(B-V) \simeq 2.1$, whilst at $E(B-V) \sim 2.3$, it begins to look as though the blue half of the line profile has been pushed high relative to the red half.

A further property of the observed spectrum is the presence of diffuse interstellar band (DIB) absorption. For example, arrayed very clearly across the top of the very same CIV $\lambda \lambda 5801,12$ feature are a number of DIBs, together with the interstellar Na I D lines (see Figure 4) perched on its redward side. We gain some insight into the physical character of the sightline to WR 93b if we compare the equivalent widths of these DIBs with their counterparts observed in the spectrum of HD 183143, a reference object for DIB studies (Herbig 1995). These data are laid out in Table 3. It can be seen that the two sets of equivalent widths are

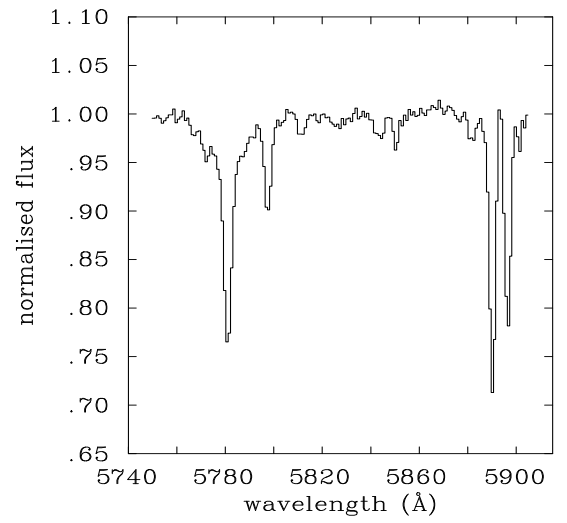

Figure 4. The diffuse interstellar bands and $\mathrm{Na}$ I $\mathrm{D}$ lines seen at high signal-to-noise ratio across the top of the C IV $\lambda \lambda 5801,12$ emission line profile. The spectrum has been rectified by fitting out the shape of the C IV emission profile (see figure 3 ).

Table 3. Equivalent width fits to DIBs located in the bright C IV $\lambda \lambda 5801,12$ emission, compared with results for the DIB reference star HD 183143 (Herbig 1995). Where no FWHM value is given it was set equal to the spectral resolution in the fit procedure. The last 3 rows also list equivalent width measurements for the wellknown interstellar $\mathrm{Na} \mathrm{I}$ and $\mathrm{K}$ I absorption lines: the reference wavelengths are laboratory values in these cases.

\begin{tabular}{llrrr}
\hline $\begin{array}{l}\lambda_{r e f} \\
(\AA)\end{array}$ & $\begin{array}{l}\lambda_{\text {obs }} \\
(\AA)\end{array}$ & $\begin{array}{r}\text { FWHM } \\
(\AA)\end{array}$ & $\begin{array}{r}\text { EW } \\
(\mathrm{m} \AA)\end{array}$ & $\begin{array}{r}\mathrm{EW}_{\text {ref }} \\
(\mathrm{m} \AA)\end{array}$ \\
\hline 5766.05 & $5766.9 \pm 0.7$ & & $38 \pm 23$ & 39 \\
5772.49 & $5771.8 \pm 0.5$ & & $60 \pm 25$ & 31 \\
5778.3 & $5781.2 \pm 0.6$ & $17.5 \pm 2.3$ & $1061 \pm 80$ & 950 \\
5780.45 & $5781.0 \pm 0.05$ & $3.4 \pm 0.2$ & $673 \pm 52$ & 801 \\
5796.98 & $5797.6 \pm 0.1$ & $3.3 \pm 0.3$ & $347 \pm 30$ & 238 \\
5809.13 & $5811.5 \pm 0.45$ & $3.5 \pm 1.0$ & $86 \pm 23$ & 34 \\
5843.6 & $5843.5 \pm 0.5$ & $4.5 \pm 1.1$ & $122 \pm 26$ & 118 \\
5849.65 & $5850.4 \pm 0.25$ & & $100 \pm 17$ & 82 \\
\hline 5889.95 & $5890.2 \pm 0.05$ & & $859 \pm 17$ & \\
5895.92 & $5896.5 \pm 0.05$ & & $670 \pm 17$ & \\
7698.98 & $7699.3 \pm 0.1$ & & $250 \pm 20$ & \\
\hline
\end{tabular}

broadly comparable, despite the fact the accepted colour excess for HD 183143 is $E(B-V)=1.3$, significantly less than determined from the SED of the WO star. (Note, in particular, that the fitting procedure, which had difficulty locating and distinguishing the broad $5778 \AA$ DIB beneath the much sharper $5780.45 \AA$ DIB, returns an equivalent width sum for these two features that differs negligibly from that measured for HD 183143.) This discrepancy amounts to a strong indication that a substantial part of the WO star reddening arises in intervening molecular gas, rather than solely in diffuse-cloud atomic gas with which the DIB column is known to correlate (see Herbig 1995 and references therein). 
Table 4. Comparison between 2MASS broadband $J, H$ and $K$ magnitudes for WR 93b and those of the LMC WO3 star, Sand 2 and Galactic WO2 stars, Sand 5. The letter in parentheses after each observed magnitude is the quality flag specified in the 2MASS database.

\begin{tabular}{|c|c|c|c|c|c|c|c|c|c|}
\hline & $A_{\lambda} / A_{V}$ & \multicolumn{2}{|c|}{$\begin{array}{l}\text { WR 93b } \\
(E(B-V)=2.1 \pm 0.1)\end{array}$} & \multicolumn{2}{|c|}{$\begin{array}{l}\text { Sand } 2 \\
(E(B-V)=0.19 \pm 0.03)\end{array}$} & \multicolumn{2}{|c|}{$\begin{array}{l}\text { Sand } 5 \\
(E(B-V)=1.7 \pm 0.1)\end{array}$} & \multicolumn{2}{|c|}{$\begin{array}{l}\text { corrected magnitude } \\
\text { differences }\end{array}$} \\
\hline$H$ & 0.190 & $10.56 \pm 0.04(\mathrm{~A})$ & $9.32 \pm 0.07$ & $15.33 \pm 0.13(\mathrm{~B})$ & $15.22 \pm 0.13$ & $8.89 \pm 0.02(\mathrm{~A})$ & $7.89 \pm 0.06$ & $5.90 \pm 0.15$ & $-1.43 \pm 0.09$ \\
\hline \multirow[t]{2}{*}{$K$} & 0.114 & $10.17 \pm 0.04(\mathrm{~A})$ & $9.43 \pm 0.06$ & $14.88 \pm 0.14(\mathrm{~B})$ & $14.82 \pm 0.14$ & $8.60 \pm 0.02(\mathrm{~A})$ & $8.00 \pm 0.04$ & $5.39 \pm 0.15$ & $-1.43 \pm 0.07$ \\
\hline & & & & & & \multicolumn{2}{|c|}{ adopted mean differences: } & $5.7 \pm 0.10$ & $-1.43 \pm 0.04$ \\
\hline
\end{tabular}

\section{ESTIMATION OF THE C/HE AND O/HE ABUNDANCE RATIOS FROM THE EMISSION LINE SPECTRUM}

We have used the recombination line method of KBS95 (their Section 8.2) to derive the relative abundances of helium, carbon and oxygen ions in the wind of WR 93b. We used the emission line equivalent widths listed in column 4 of Table 2 to calculate the dereddened line fluxes, $I$, in column 5 , by assuming that the underlying continuum follows a $\mathrm{F}_{\lambda} \propto \lambda^{-3}$ spectrum (normalised at $6000 \AA$ to $1.30 \times 10^{-13}$ ergs $\left.\mathrm{cm}^{-2} \mathrm{~s}^{-1} \AA^{-1}\right)$. The adopted line emission coefficients, $Q\left(=h \nu \alpha_{r e c}\right)$, were taken from Table 14 of KBS95. The ratio $I / Q$, given in column 6 of Table 2 is linearly proportional to ion abundance.

It is safe to assume that the overwhelmingly dominant ion stage of carbon in the wind of WR $93 \mathrm{~b}$ is $\mathrm{C}^{4+}$, and that helium is ionized to $\mathrm{He}^{2+}$. Following KBS95, we use the isolated C IV 9-7 $7062 \AA$ line as the preferred diagnostic for the $\mathrm{C}^{4+}$ abundance, to obtain an $I / Q$ of $1.35 \times 10^{14} \mathrm{~cm}^{-5}$. We used this $I / Q$ to then estimate the fraction (45\%) of the $6560 \AA$ feature flux contributed by C IV $12-8$ and $7 \mathrm{p}-5 \mathrm{~s}$. The remaining $55 \%$ of the $6560 \AA$ flux could then be attributed to He II 6-4: on this basis, an $I / Q$ of $1.42 \times 10^{14} \mathrm{~cm}^{-5}$ was derived for $\mathrm{He}^{2+}$.

Unlike carbon, oxygen can be expected to be and indeed is observed to be distributed across a number of ion stages. The isolated O vi lines at $5290 \AA$ (8-7) and $6200 \AA$ (11-9,13-11) yielded reassuringly similar $I / Q$ values for $\mathrm{O}^{6+}$ of $1.05 \times 10^{13} \mathrm{~cm}^{-5}$ and $1.08 \times 10^{13} \mathrm{~cm}^{-5}$, respectively. For the other recombining oxygen ion seen prominently in the spectrum, $\mathrm{O}^{5+}$, we use the O v $5590 \AA 3 \mathrm{~d}-3 \mathrm{p}$ emission to determine an $I / Q$ of $6.3 \times 10^{13} \mathrm{~cm}^{-5}$. Finally, in order to fit the weak, very high excitation, $6070 \AA$ feature we assumed, like KBS95, that the abundances of (and hence $I / Q$ values for) $\mathrm{O}^{7+}$ and $\mathrm{O}^{8+}$ were the same, on which basis the $\mathrm{O}$ VII 12-10 transition at $6085 \AA$ is found to contribute $15 \%$ of the total flux, with the O viII 10-9, 13-11 lines at 6068,6064 $\AA$ contributing the remainder: for both ions, $I / Q=4.3 \times 10^{11}$ $\mathrm{cm}^{-5}$.

Summing all ionic species, we find $\mathrm{C} / \mathrm{He}=0.95, \mathrm{O} / \mathrm{He}$ $=0.13$ and $(\mathrm{C}+\mathrm{O}) / \mathrm{He}=1.08$, by number. The $\mathrm{O} / \mathrm{He}$ ratio of 0.13 derived for WR $93 \mathrm{~b}$ is similar to the values (0.100.11 , by number) derived by KBS95 for the LMC WO3 star Sand 2 and for the Galactic WO2 stars Sand 4 and Sand 5. However, the $\mathrm{C} / \mathrm{He}$ ratio of 0.95 for $\mathrm{WR} 93 \mathrm{~b}$ is sigificantly higher than the $\mathrm{C} / \mathrm{He}$ ratios of 0.51-0.52 derived for these other WO stars. Expressed as mass fractions the abundances obtained here for WR 93b are $\mathrm{X}(\mathrm{He})=0.23, \mathrm{X}(\mathrm{C})=0.65$ and $\mathrm{X}(\mathrm{O})=0.12$.

\section{THE DISTANCE TO WR 93B}

A means to estimating the distance to the newly-discovered WO star is to apply the estimated total visual extinction in combination with a scaling to the reddening-corrected magnitudes of the WO3 star, Sand 2, and WO2 star, Sand 5 . For both comparison stars - so close in spectral type to that of the new WO star - reliable reddenings and distances can be derived from the literature: Sand 2 is in the LMC with a modest reddening of $E(B-V)=0.19$ (Crowther et al 2000), whilst Sand 5 is located in the open cluster Berkeley 87, in the Cygnus X region of the Galaxy, behind a reddening of $E(B-V)=1.7 \pm 0.1$ (Turner \& Forbes 1982). We must assume, of course, that WR 93b, Sand 2 and Sand 5 share similar intrinsic stellar and spectral properties. It is an encouraging start that the continuum $M_{V}$ s for these references objects are indeed almost the same: for Sand 2, $M_{V}=-3.0$ (Crowther et al 2000), whilst for Sand $5, M_{V}=$ -3.1 for $V=13.37$ (KBS95) at $1.75 \mathrm{kpc}$ (see below).

To minimise the impact of reddening uncertainties on this comparison, it can be performed in the nearinfrared using data taken from the 2MASS database (see http://www.ipac.caltech.edu/2mass/). The 2MASS JHK magnitudes for the three stars are set out in Table 4 . Using the tabulation of $A_{\lambda} / A_{V}$ given by Cardelli, Clayton \& Mathis (1989) and the available visual extinctions we correct the NIR magnitudes to zero reddening. A little experimentation with the value of $E(B-V)$ for WR $93 \mathrm{~b}$ reveals that $E(B-V) \simeq 2.1$ is again favoured as it yields dereddened NIR magnitudes that are most uniformly offset from the high quality photometry of Sand 5. The consistency of outcome with respect to Sand 2 for this same reddening is also satisfactory given the larger photometry errors reported for this object. We find, after applying the reddening corrections, that the new WO star is $\sim 5.7$ magnitudes brighter than Sand 2, and fainter than Sand 5 by $\sim 1.43$ magnitudes.

Adopting a distance of $49 \mathrm{kpc}$ to the LMC (see e.g.Gibson 2000), the difference of 5.7 magnitudes with respect to Sand 2 yields a distance of $3.5 \mathrm{kpc}$ to WR $93 \mathrm{~b}$. Two recent estimates of the distance to Be 87, the cluster hosting Sand 5, are respectively $1.6 \mathrm{kpc}$ (Massey, DeGioiaEastwood \& Waterhouse 2001) and $1.9 \mathrm{kpc}$ (Knödlseder et al 2002): we adopt the intermediate value of $1.75 \mathrm{kpc}$. The NIR magnitude difference of -1.43 , combined with this distance then yields a distance to WR $93 \mathrm{~b}$ of $3.4 \mathrm{kpc}$. This 
Table 5. The names, spectral types, Galactic co-ordinates and distances to the known Galactic WO stars, incorporating the newly discovered WO star, WR 93b. The spectral types for all but WR 93b are from Crowther et al (1998): that for WR93b has been estimated here using the same criteria. Two distances are given: the first $\left(D_{\odot}\right)$ is the derived distance from the Sun, while the second $\left(D_{G C}\right)$ is the distance from the Galactic Centre. The calculation of the latter assumes the Sun is at a radius of 7.94 kpc (Eisenhauer et al 2003) and neglects the small angular displacement of each WO star out of the Galactic equatorial plane. The references in the final column are the sources for the heliocentric distance in each case.

\begin{tabular}{|c|c|c|c|c|c|c|c|}
\hline Object & $\begin{array}{l}\text { WR catalogue } \\
\text { number }\end{array}$ & spectral type & $\begin{array}{c}\text { Gal. } \\
\ell^{\circ}\end{array}$ & $\begin{array}{r}\text { co-ords. } \\
b^{\circ}\end{array}$ & $\begin{array}{r}D \odot \\
(\mathrm{kpc})\end{array}$ & $\begin{array}{l}D_{G C} \\
(\mathrm{kpc})\end{array}$ & Distance reference \\
\hline \multirow[t]{2}{*}{ MS 4} & WR 30a & $\mathrm{WO} 4+\mathrm{O} 5$ & 288.90 & -1.38 & 7.8 & 9.2 & van der Hucht 2001 \\
\hline & WR 93b & WO3 & 353.27 & -0.85 & 3.4 & 4.6 & this paper \\
\hline Sand 4 & WR 102 & WO2 & 2.38 & +1.41 & 4.6 & 3.4 & this paper \\
\hline Sand 5 & WR 142 & WO2 & 75.73 & +0.30 & 1.8 & 7.7 & $\begin{array}{l}\text { Be } 87 \text { cluster: Massey et al 2001, } \\
\text { Knödlseder et al } 2002\end{array}$ \\
\hline
\end{tabular}

Table 6. Summary list of the parameters of WR $93 \mathrm{~b}$

\begin{tabular}{lll}
\hline Parameter & value & comment \\
\hline Position (RA,Dec) & $173203.30-350432.5$ & J2000, from SHS database \\
$R, I$ magnitudes & $14.7,12.5$ & from SHS database \\
$J, H, K$ magnitudes & $11.33 \pm 0.03,10.56 \pm 0.04,10.17 \pm 0.04$ & from 2MASS database \\
$\mathrm{E}(B-V)$ & $2.1 \pm 0.1$ & $R=3.1$ gives $\mathrm{A}_{V} \simeq 6.5$ \\
spectral type & $\mathrm{WO} 3$ & criteria place WR 93b closer \\
& & to WO2/3 boundary than Sand 2 \\
wind terminal velocity & $5750 \mathrm{~km} \mathrm{~s}^{-1}$ & \\
abundance ratios: & $\mathrm{C} / \mathrm{He}=0.95$ & by number \\
& $\mathrm{O} / \mathrm{He}=0.13$ & " " \\
distance & $(\mathrm{C}+\mathrm{O}) / \mathrm{He}=1.08$ & \\
\hline
\end{tabular}

is very satisfying agreement. But, given the systematic uncertainty contained within the assumption of shared stellar parameters, we should anticipate an error on $3.4 \mathrm{kpc}$ of up to $10 \%$ (equivalent to $\sim 0.2$ offset in $M_{V}$ ).

The Galactic co-ordinates of WR 93b are $\ell=353.27^{\circ}$, $b=-0.85^{\circ}$, placing it less than $7^{\circ}$ from the Galactic Centre line of sight, and outside the strips surveyed for Wolf-Rayet stars by Shara et al (1999). At a distance of $3.4 \mathrm{kpc}$ the new WO star would be located very close to the structure Russeil (2003) identifies as the Scutum-Crux arm, well inside the long-established Sagittarius-Carina arm at $\sim 2 \mathrm{kpc}$ in the same direction. For a necessarily young object like a Wolf-Rayet star this is a perfectly acceptable place to be. Projected onto the plane of the sky, the nearest H II region (a signature of continuing star formation) listed by Russeil (2003, Table 3) is about half a degree away from WR 93b at $\ell=353.43^{\circ}, b=-0.368^{\circ}$. At $3.4 \mathrm{kpc}$ this angular separation converts to a length of about $30 \mathrm{pc}$. The kinematic distance to this HiI region, which is 'compact' and without a known optical counterpart, has been estimated from Caswell \& Haynes' (1987) measurement of its radio recombination line radial velocity. In the framework adopted by Russeil (2003), wherein the Galactic Centre is $8.5 \mathrm{kpc}$ away, the distance to this $\mathrm{H}$ II region is required to be $3.5_{-0.7}^{+0.6} \mathrm{kpc}$. This is consistent with our estimate of the distance to the WO star. If the Galactic Centre is taken to be $7.94 \pm 0.42 \mathrm{kpc}$ as determined recently by Eisenhauer et al (2003), this revises the kinematic distance downwards a little to $3.3 \mathrm{kpc}-$ leaving the quality of agreement unchanged.

\section{DISCUSSION}

With the addition of this newly-revealed WO star, the elite club of known Galactic WO stars has increased its membership to four. We consider briefly how these stars are distributed within the disk of the Milky Way. In Table 5 we list the Galactic co-ordinates and estimated distances of all 4 stars and use these data to calculate their Galacto-centric distances. The distance from the Sun to MS 4 (WR 30a) is adopted from van der Hucht (2001), which in turn is based on the absolute calibration of O stars due to Vacca, Garmany \& Shull (1996). The distance to Sand 4 (WO2, also known as WR 102) is particularly uncertain, with literature estimates ranging from $2.3 \mathrm{kpc}$ (Kingsburgh et al 1995) up to $5.6 \mathrm{kpc}$ (van der Hucht 2001). Here we have derived and use a value of $4.6 \mathrm{kpc}$ on the basis of the same IR photometric scaling relative to Sand 5 (also WO2) that we have used to determine the distance to WR 93b. This object joins WR 93b in lying far inside the solar circle, whilst the other two WO stars, Sand 5 (WR 142) and MS 4 lie at Galactocentric radii similar to that of the Sun.

The existence of this small grouping, taken on its own, would not encourage the idea that evolution to a longerlived WO stage is favoured in regions of reduced metallicity (Maeder 1991). Nevertheless it is a fact that the three WO stars located in other galaxies are all found in low metallicity environments (the LMC, SMC and IC 1613), as predicted by stellar evolutionary models such as those of Maeder (1991). If theory is qualitatively correct, the conclusion must be that a Milky Way WO star population at large Galactocentric radii may still be waiting to be discovered. This remains 
a distinct possibility because the northern Galactic Plane, which includes the anti-centre region has not yet been surveyed systematically for Wolf-Rayet stars of any sub-type. By contrast the southern Plane has been better served, both by the Stephenson \& Sanduleak (1971) objective prism survey and, most recently, by Shara et al (1999) - and of course by the AAO/UKST H $\alpha$ survey exploited here.

An alternative phenomenological view of this still modest Galactic WO star population is that, as higher excitation, extreme examples of early WC stars (see e.g. Crowther et al 1998), they should follow a similar trend with respect to Galactocentric radius to that exhibited by their less elusive relations. If it should turn out that the WO stars are more frequent inside the solar circle than outside it, this would amount to the same trend as is now recognised for WC stars by e.g. Massey \& Johnson (1998) and Shara et al (1999).

In this paper we have reported the bare facts on a new massive Galactic WO3 star (the only example of its subtype in the Milky Way - they are so few). Our quantitative findings are summarised in Table 6 . It seems that this is a star with a record-holding stellar wind: no other wind from a non-degenerate star in the Galaxy is seen to attain so high a terminal velocity $\left(5750 \mathrm{~km} \mathrm{~s}^{-1}\right)$. It is also the most highly reddened WO star discovered so far, with $A_{V} \simeq 6.5$. Last we note that WR 93b is remarkable also with regard to its carbon abundance relative to helium: no other known Wolf-Rayet star can match $\mathrm{C} / \mathrm{He}=0.95$ by number. Among the WO stars, only Sand 1 in the SMC approaches such an extreme ratio with $\mathrm{C} / \mathrm{He}=0.81$ (KBS95). But there the similarity ends in that, in Sand $1, \mathrm{O} / \mathrm{He}$ is also significantly enhanced relative to the other $\mathrm{WO}$ stars $(\mathrm{O} / \mathrm{He}=0.3$, as compared with $\sim 0.1$ ), while it is not in WR 93b. As is often the case in the presence of such a sparse sample of objects, the emergent picture from - in this instance - the known abundance patterns is too poorly formed to allow any astrophysical conclusions to be drawn. The clear need is to find more of them. We end on the optimistic note, that relative to the power of today's spectroscopic capabilities, WR $93 \mathrm{~b}$ at $R \simeq 14.7$ is a bright object: appropriate spectroscopic surveys of the Galactic Plane are bound to find more.

\section{ACKNOWLEDGMENTS}

We would like to thank Nigel Hambly for valuable help on many occasions in connection with the exploitation of the SHS database. MP and RW both acknowledge the support of postgraduate studentships funded by the Particle Physics \& Astronomy Research Council of the United Kingdom. This paper makes use of: data obtained with the AAO/UK Schmidt Telescope at Siding Spring Observatory, NSW, Australia; data from the William Herschel Telescope, operated on the island of La Palma by the Isaac Newton Group in the Spanish Observatorio del Roque de los Muchachos of the Instituto de Astrofisica de Canarias; data products from the Two Micron All Sky Survey, which is a joint project of the University of Massachusetts and the Infrared Processing and Analysis Center/California Institute of Technology, funded by the National Aeronautics and Space Administration and the National Science Foundation.

\section{REFERENCES}

Barlow M.J., Hummer D.G., 1982, in IAU Symp. 99, Wolf-Rayet Stars: Observations, Physics, Evolution, eds. C.W.H. deLoore \& A.J. Willis, Dordrecht:Reidel, p387

Cardelli J.A., Clayton G.C., Mathis J.S., 1989, ApJ, 345, 245

Caswell J.1., Haynes R.F., 1987, A\&A, 171, 261

Crowther P. A., de Marco O., Barlow M. J., 1998, MNRAS, 296, 367

Crowther P. A., et al., 2000, ApJ, 538, L51

Eisenhauer F., Schödel R., Genzel R., Ott T., Tecza M., Abuter R., Eckart A., Alexander T., 2003, ApJ, 597, L121

Gibson B.K., 2000, MmSAI, 71, 693

Hambly N.C., Irwin M. J., MacGillivray H. T., 2001, MNRAS, 326,1295

Herbig G.H., 1995, ARA\&A, 33, 19

Kingsburgh R.L., Barlow M.J., 1995, A\&A, 295, 171

Kingsburgh R. L., Barlow M. J., Storey P. J., 1995 A\&A, 295, 75 (KBS95)

Knödlseder J., Cerviño M., Le Duigou J.-M., Meynet G., Schaerer D., van Ballmoos P., 2002, A\&A, 390, 945

MacConnell D.J., 1982, A\&AS, 48, 355

MacConnell D.J., Sanduleak N., 1970, PASP, 82, 80

Maeder A., 1991, A\&A, 242, 93

Massey P., DeGioia-Eastwood K., Waterhouse E., 2001, AJ, 121, 1050

Massey P., Johnson O. 1998, ApJ, 505, 793

Massey P., Strobel K., Barnes J. V., Anderson E., 1988, ApJ, 328, 315

Morgan D.H., Parker Q.A., Russeil D., 2001, MNRAS 322, 877

Morris P.W., Brownsberger K.R., Conti P.S., Massey P., Vacca W.D., 1993, ApJ, 412, 324

Parker Q.A., Bland-Hawthorn J., 1998, PASA, 15, 33

Parker Q.A., Phillipps S., 1998, PASA 15, 28

Parker Q.A., Malin D.F., 1999, PASA 16, 288

Parker, Q.A \& Morgan, D.H., 2003, MNRAS, 341, 961

Parker, Q.A. et al. 2003, in Dopita M.A., Kwok S., Sutherland R., eds, Proc. IAU Symp. 209, Planetary Nebulae, Astronomical Society of the Pacific (pub), p25

Robertson T.H., Jordan T.M., 1989, AJ, 98, 1354

Russeil D, 2003, A\&A, 397, 133

Sanduleak N., 1971, ApJ, 164, L71

Schaefer B.E., et al., 2003 ApJ, 588, 387

Shara M. M., Moffat A. F. J., Smith L. F., Niemela V. S., Potter M., Lamontagne R., 1999, AJ, 118, 390

Smith L.F., Shara M.M., Moffat A.F., 1990, ApJ, 358, 229

Stephenson C. B., Sanduleak N., 1971 PW\&SO 1, 1

Turner D.G., Forbes D., 1982, PASP, 94, 789

Vacca W. D., Garmany C. D., Shull J. M., 1996, ApJ, 460, 914

van der Hucht, K. A., 2001, NewAR, 45, 135

Woosley S.E., Heger A., Weaver T.A., 2002 RvMP, 74, 1015 